\title{
Long-Term Effects of Brief Antihypertensive Treatment on Systolic Blood Pressure and Vascular Reactivity in Young Genetically Hypertensive Rats
}

\author{
Oren Traub, Mary C. Lloyd, \\ and $R$. Clinton Webb \\ Department of Physiology, The University of Michigan, \\ Ann Arbor, Michigan
}

\begin{abstract}
Summary. Recent studies have shown that angiotensin converting enzyme (ACE) inhibitor treatment in young spontaneously hypertensive rats (SHR) reduces blood pressure into adulthood. This study explored changes in vascular reactivity in adult normotensive (WKY) rats and stroke-prone SHR (SHRSP) receiving the following treatments at 6-10 weeks of age: (a) ACE inhibitor (ramipril); (b) hydralazine/hydrochlorothiazide (hydral/HCTZ); or (c) no treatment. The hypothesis tested was that vascular changes and blood pressure would be reduced in adult SHRSP treated with ramipril during development. At 17 weeks of age, rats were anesthetized and vascular tissue was excised. Isolated experiments in the aorta included characterization of initial phasic and tonic contractions to $0.1 \mu \mathrm{M}$ angiotensin II (AII). A phenylephrine (PE) concentration-response curve was performed on carotid arteries, and threshold values were determined. All WKY groups showed lower systolic blood pressure $(131 \pm 4 \mathrm{mmHg})$ and reduced phasic AII induced contraction $(7.4 \pm 4.7 \%)$ compared with SHRSP $(217 \pm 4 \mathrm{mmHg} ; 37.2 \pm 4 \%)$. Antihypertensive treatment reduced blood pressure (ramipril: $168 \pm$ 2; hydral/HCTZ: $198 \pm 6 \mathrm{mmHg}$ ) but not phasic AII responses in adult SHRSP; adult WKY rats were unaffected by treatment. Threshold values for $P E$ in carotid arteries were lower in SHRSP than in WKY, indicating increased sensitivity. However, SHRSP treated with ramipril did not demonstrate increased sensitivity to $P E$. These data support the hypothesis that blood pressure and sensitivity to $P E$ but not contractile responsiveness to AII in adult SHRSP are determined by an AII-sensitive mechanism during development.
\end{abstract}

Cardiovasc Drugs Ther 1995;9:421-429

Key Words. ACE inhibitors, adrenergic, angiotensin II, antihypertensive, genetic hypertension, ramipril, spontaneously hypertensive rats, vascular smooth muscle

Current evidence suggests that hereditary factors play an important role in the enhanced vascular reactivity in genetic hypertension $[1,2]$. Several recent studies have shown increased vascular responsiveness to agonists $[3,4]$ in genetically hypertensive rats (SHR). Further, several of these enhanced responses were associated with elevated blood pressure [5].

Recent investigations have focused on development aspects of hypertension. Specifically, the role that the renin-angiotensin system plays in the expression of genetic hypertension has come under scrutiny. Several studies have shown that SHR treated with an angiotensin converting enzyme (ACE) inhibitor during a so-called critical period of development (2-10 weeks of life) do not elevate arterial blood pressure to the same magnitude as that observed in untreated SHR or in SHR treated with the ACE inhibitor during a noncritical period [6-10]. It has been postulated that these effects may be due to prevention of gene switching. This switching appears to represent an adaptive response to recruit genes that are normally active only during fetal development and that code for proteins that are responsible for the maintenance of elevated blood pressure in adulthood. These studies, however, failed to characterize vascular responses to agonists that have been observed to be different in SHR in comparison with normotensive (WKY) rats. The goals of the present study were as follows: (1) to examine vascular responses in stroke-prone SHR (SHRSP) that differ from WKY and how these traits are affected by blocking the generation of angiotensin II (AII) during development; and (2) to control for the direct effect of lower blood pressure during the critical period by using a non-angiotensin-dependent antihypertensive treatment. The hypotheses tested were that abnormal vascular responses and blood pressure may be reduced in adult SHRSP treated with ramipril at an early age and that reversal of the vascular abnormalities in hypertension may be independent of the blood pressure-lowering effect of ACE inhibitors.

\section{Materials and Methods}

\section{Animal treatment}

Male SHRSP and WKY rats were obtained from a colony maintained in the Department of Anatomy and

Address for correspondence: Oren Traub, Department of Physiology, The University of Michigan, 7813 Medical Science Building II, Ann Arbor, MI 48109-0622.

Received 14 June 1994, accepted in revised form 1 November 1994 
Cell Biology at the University of Michigan (Ann Arbor, MI). All rats were housed in a light-cycled, temperature-controlled room with ad libitum access to food and water. At all times excluding the 6-10 week age period, rats received tap water and standard laboratory chow (Purina Mills Co., Richmond, IN). Body weight was recorded and systolic blood pressure was measured weekly. Systolic blood pressure was measured by an indirect tail cuff (pneumatic transducer) method in conscious, restrained rats. Three experimental treatment regimens were included in this protocol: untreated $(\mathrm{WKY}, \mathrm{n}=8$; SHRSP, $\mathrm{n}=8$ ), ACE inhibitor treated (WKY, $\mathrm{n}=$ 8; SHRSP, $\mathrm{n}=8$ ), and diuretic/vasodilator treated (WKY, $\mathrm{n}=6$; SHRSP, $\mathrm{n}=6$ ). One untreated WKY and one SHRSP diuretic/vasodilator-treated rat did not survive the study and were excluded from the analysis. Rats in the diuretic/vasodilator group received hydralazine $(200 \mathrm{mg} / \mathrm{l})$ and hydrochlorothiazide $(200 \mathrm{mg} / \mathrm{l})$ in their drinking water during the treatment period. Based on an intake of $20 \mathrm{ml}$ of water per day, rats received $4 \mathrm{mg} /$ day of each drug. All other rats received tap water. Rats in the ACE inhibitortreated group were given ramipril in their food ( $0.0025 \%$ ramipril by weight) from 6 to 10 weeks of age. Measured dosages of ramipril were WKY, 2.94 $\pm 0.16 \mathrm{mg} / \mathrm{kg} /$ day and SHRSP $3.23 \pm 0.18 \mathrm{mg} / \mathrm{kg} /$ day $(p>0.05)$. All other rats received control food.

\section{Experimental protocol}

At 17 weeks of age, rats were anesthetized with sodium pentobarbital $(50 \mathrm{mg} / \mathrm{kg})$ and decapitated. Thoracic aortae and carotid arteries were harvested and placed in cold physiological salt solution (PSS composition in $\mathrm{mmol} / \mathrm{l}$ was as follows: $\mathrm{NaCl} 130, \mathrm{KCl} 4.7$, $\mathrm{KH}_{2} \mathrm{PO}_{4} 1.18, \mathrm{MgSO}_{4} \cdot 7 \mathrm{H}_{2} \mathrm{O}$ 1.17, $\mathrm{NaHCO}_{3} 14.9$, dextrose 5.5, $\mathrm{CaNa}_{2}$ EDTA $0.026, \mathrm{CaCl}_{2} 1.6$ ) for $\sim 20$ hour storate. Preliminary experiments showed that this storage period did not significantly alter responses in arteries from either SHRSP or WKY rats. Fatty and connective tissue was removed from the thoracic aorta and carotid arteries, and the vessels were cut into helical strips ( $2 \mathrm{~mm} \times 15 \mathrm{~mm}$ for aorta; $0.7 \mathrm{~mm} \times 10$ $\mathrm{mm}$ for carotid arteries). The endothelium was removed by gentle rubbing of the intima with a cotton swab. Functional removal of the endothelium was confirmed by lack of relaxation to acetylcholine after a phenylephrine-induced contraction. Vascular strips were mounted on stainless steel tissue hangers and suspended in a muscle bath containing PSS maintained at $37^{\circ} \mathrm{C}$ and aerated with $95 \% \mathrm{O}_{2}$ and $5 \% \mathrm{CO}_{2}$. Strips were allowed to equilibrate for 90 minutes with a passive force of 1.5 and $0.6 \mathrm{~g}$ for aorta and carotid arteries, respectively. Drugs were added directly to the bath and isometric contractile force was measured using Grass FT.03 force transducers and was recorded on a Grass polygraph. Between responses, agonists were rinsed from the tissue bath and strips were allowed to recover to baseline values. Contrac- tile responses to angiotensin II were measured in thoracic aorta. Cumulative concentration-response curves to phenylephrine were generated using carotid arteries.

Plasma renin activity assays were conducted on blood samples taken from anesthetized rats according to the method described by Sessler et al. [11]. Aliquots of incubation media, plasma, homogenates, and gel elutions were combined with substrate prepared from 40 hour nephrectomized rats. After incubation, the angiotensin I generated was measured using a radioimmunoassay kit (New England Nuclear, Boston, MA).

\section{Drugs}

Angiotensin II, hydralazine hydrochloride, and hydrochlorothiazide were purchased from Sigma Chemical (St. Louis, MO). Phenylephrine hydrochloride was obtained from the University of Michigan Hospital Pharmacy (Ann Arbor, MI), and Ramipril was the generous gift of the Upjohn Co. (Kalamazoo, MI). Laboratory chow containing $0.0025 \%$ ramipril by weight was prepared for this study by Purina Mills (Richmond, IN). Hydralazine and hydrochlorothiazide were prepared in tap water. All other drugs were prepared and diluted in deionized water. Other chemicals used in this study were of reagent grade.

\section{Statistical analysis}

Data are expressed as mean \pm standard error of the mean (SEM). Unpaired Student's $t$ tests were used to determine statistical significance between SHRSP and WKY rats and within groups. A $p$ value $<0.05$ was considered significant. The Bonferroni correction was applied to adjust for multiple comparisons. $\mathrm{EC}_{10}$ values were determined following graphical representation of normalized concentration-response curves. Blood pressure correlation with vascular responses were calculated using linear regression analysis.

\section{Results}

Rats were treated with hydral/HCTZ or ramipril from 6 to 10 weeks of age, and blood pressures and weights were measured until 17 weeks of age. Control rats received no treatment. Figure 1 illustrates the effect of the antihypertensive treatments on the time course of systolic blood pressure and weight development in genetically normotensive and hypertensive rats. As seen in Figure 1, both antihypertensive regimens were effective in lowering systolic blood pressure in both WKY (panel A) and SHRSP (panel B) during the treatment period. After cessation of the treatments, systolic blood pressures of the WKY rats gradually approached control levels. At 16.5 weeks of age there was no statistical difference in systolic blood pressure among WKY rat groups. SHRSP animals treated with hydral/HCTZ showed an increase in systolic blood pressure after cessation of the treatment; 

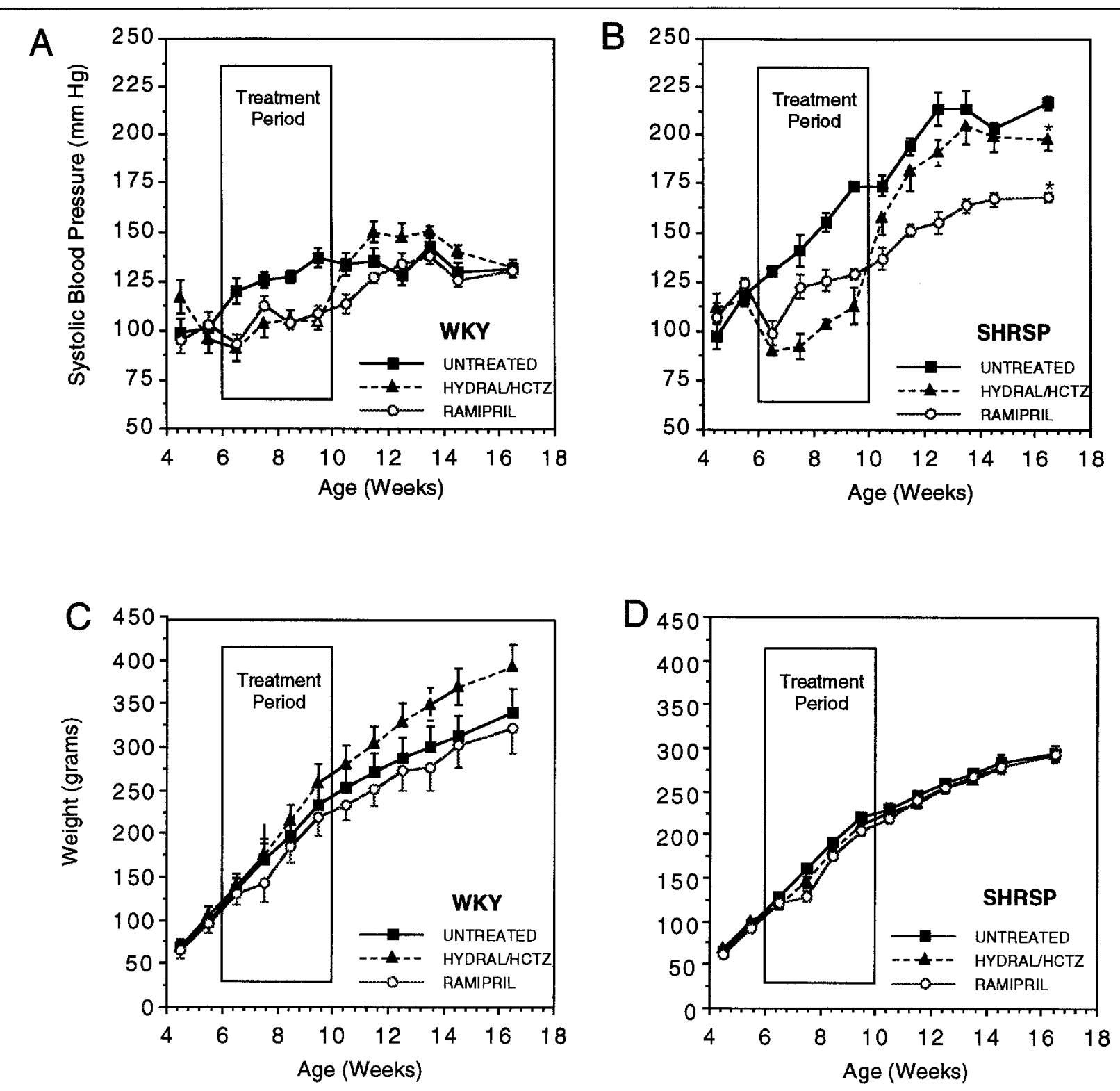

Fig. 1. Antihypertensive regime effects on blood pressure and body weight. A: Line graph showing indirect systolic blood pressure of WKY rats 4.5-16.5 weeks of age receiving no treatment $(n=7)$, hydralazine/HCTZ treatment $(n=6)$, or ramipril treatment $(n=8)$. B: Line graph showing indirect systolic blood pressure of SHRSP rats 4.5-16.5 weeks of age receiving no treatment $(n=$ 8), hydralazine/HCTZ treatment $(n=5)$, or ramipril treatment $(n=8)$. C: Line graph showing body weight of WKY rats 4.5-16.5 weeks of age receiving no treatment $(n=7)$, hydralazine $/ H C T Z$ treatment $(n=6)$, or ramipril treatment $(n=8)$. D: Line graph showing body weight of SHRSP rats 4.5-16.5 weeks of age receiving no treatment $(n=8)$, hydralazine/HCTZ treatment $(n=5)$, or ramipril treatment $(n=8)$. All data are reported as means $\pm S E M$ values. Data points at age 15.5 weeks were excluded due to technical reasons. ${ }^{*} p<.01$ compared to untreated group by test in week 16.5 of blood pressures and at all data points in body weight measurements. 
at 14.5 weeks of age there was no significant difference between the hydral/HCTZ-treated SHRSP and the untreated SHRSP, while at 16.5 weeks of age there was a significant difference (198 \pm 6 vs. $217 \pm$ $3 \mathrm{mmHg}$ ), though not to the magnitude of the ramipril-treated SHRSP. Ramipril-treated SHRSP did not show as great a rise in blood pressure, and systolic blood pressure remained significantly lower than untreated levels at 16.5 weeks of age (168 \pm 2 vs. 217 $\pm 3 \mathrm{mmHg}$ ). The systolic blood pressures of the ramipril-treated SHRSP, however, were still significantly higher than those in WKY (131 $\pm 4 \mathrm{mmHg})$. The time course for weight gain showed no statistical differences among treatments in SHRSP or WKY, although there was a trend towards higher body weights in WKY animals that received hydral/HCTZ versus control WKY animals and lower body weights in ramipriltreated WKY animals versus untreated controls.

Figure 2 illustrates the time course of force development in aortic strips exposed to $0.1 \mu \mathrm{M}$ AII. Following addition of AII to the muscle bath, aortic responses were characterized by a sharp, rapid "phasic" response that was particularly pronounced in SHRSP. Following this phasic response there was a more gradual contractile response and maintenance of tone. The maximal force development or peak response was measured as the greatest trension observed during this tonic phase. Marked oscillatory contractile activity was occasionally observed in SHRSP but never in WKY rats. Because of the low frequency of this event, however, it was not possible to determine whether either antihypertensive treatment affected this phenomenon. A single, maximal concentration of AII was utilized because of the tachyphylactic properties of AII on contractile responsiveness in rat arteries.

Peak responses to AII were not significantly different among any of the groups except untreated WKY (untreated WKY, $224 \pm 38 \mathrm{mg}$; hydral/HCTZ-treated WKY, $320 \pm 55 \mathrm{mg}$; ramipril-treated WKY, $401 \pm$ $36 \mathrm{mg}$; untreated SHRSP, $352 \pm 33 \mathrm{mg}$; hydral/

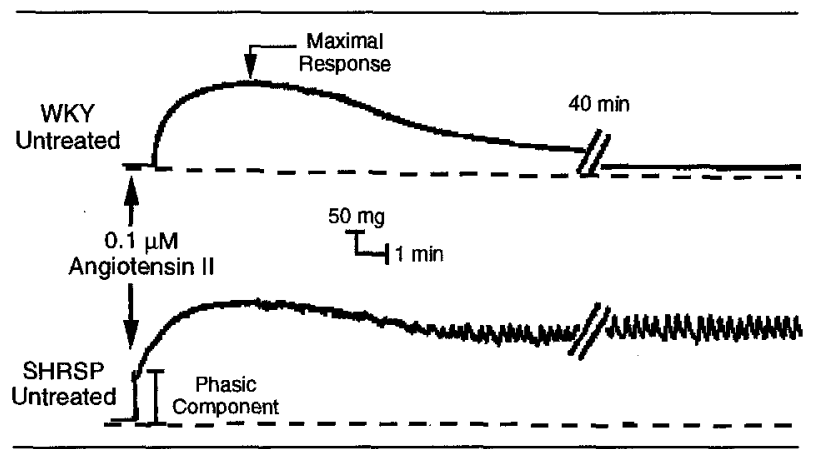

Fig. 2. Representative tracing of force development in response to angiotensin $I I(0.1 \mu M)$ in thoracic aorta from WKY (top) and SHRSP (bottom) rats. Responses were characterized by total milligrams of force development and phasic contractile response.

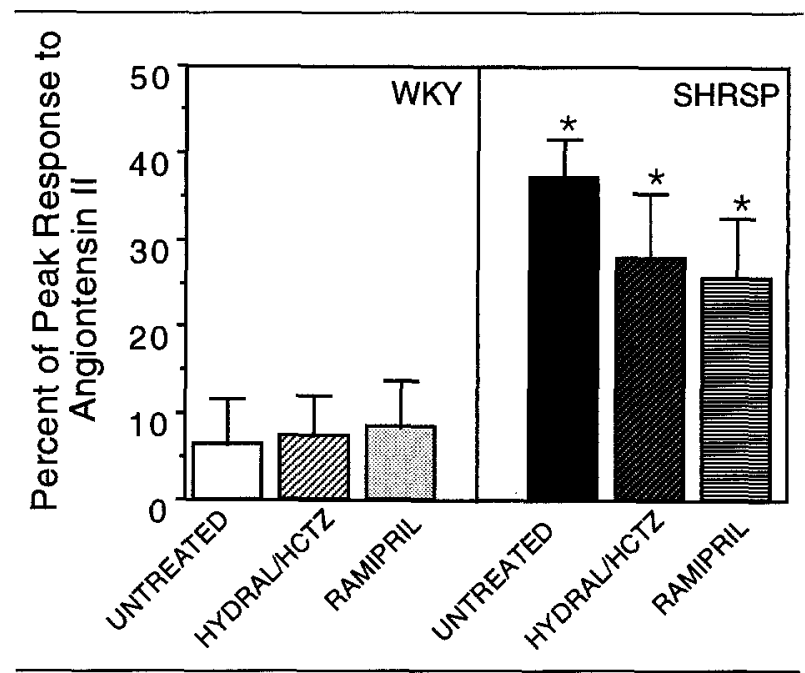

Fig. 3. Profile of contractile responses to $0.1 \mu M$ angiotensin II. Phasic response to angiotensin II in WKY and SHRSP control and treated rats. Values are expressed as percent of peak response to angiotensin II. All data are expressed as means $\pm S E M$ values. $n$ values: WKY untreated (7), WKY hydralazine/HCTZ (6), WKY ramipril (8), SHRSP untreated (8), SHRSP hydralazine/HCTZ (5), SHRSP ramipril (8). *p $<.05$ compared with untreated WKY by Student's test.

HCTZ-treated SHRSP, $354 \pm 70 \mathrm{mg}$; ramipriltreated SHRSP, $388 \pm 51 \mathrm{mg}$ ).

Figure 3 illustrates phasic contractile responsiveness to $0.1 \mu \mathrm{M}$ AII in aortic segments from all WKY and SHRSP groups. Contractile responsiveness to angiotensin II was characterized because of its relationship to the experimental intervention (ACE inhibitor). According to the hypothesis that inhibition of angiotensin II generation during development would have long-term effects, it would logically follow that some alteration in responsiveness to AII in adulthood may occur. The phasic component of contraction in vascular smooth muscle corresponds to calcium release from intracellular stores. It has previously been observed that this phasic response in response to certain agonists is greater in arteries from SHRSP than in arteries from WKY rats [12]. Phasic responses elicited by AII were easily characterized in aortae from the treated and untreated SHRSP and WKY rats because of the distinct separation between the phasic and tonic portions of contraction (Figure 3). This difference in phasic contractile responsiveness was studied with the hypothesis that ACE inhibition during development would reverse this increase in phasic contractile responsiveness to AII in aortae from SHRSP.

In all groups, aorta from SHRSP animals showed significantly greater levels of phasic contractile response to AII than WKY animals. Antihypertensive treatment had no significant effect on phasic contractile responses in the WKY or SHRSP groups. Both treatments tended to cause a decrease in phasic re- 
sponse from untreated levels in SHRSP, but this decrease was not statistically significant. However, this may be due to insufficient statistical power rather than a lack of physiological difference. Plasma renin activity was not significantly different among animal groups (untreated WKY, $23.9 \pm 2.5 \mathrm{ng} / \mathrm{ml} / \mathrm{hr}$; hydral/ HCTZ-treated WKY, $28.0 \pm 4.6 \mathrm{ng} / \mathrm{ml} / \mathrm{hr} ; \mathrm{rami}-$ pril-treated WKY, $27.8 \pm 3.1 \mathrm{ng} / \mathrm{ml} / \mathrm{hr} ; \quad$ untreated SHRSP, $26.0 \pm 1.8 \mathrm{ng} / \mathrm{ml} / \mathrm{hr}$; hydral $/ \mathrm{HCTZ}$ treated SHRSP, $30.7 \pm 0.8 \mathrm{ng} / \mathrm{ml} / \mathrm{hr} ; \mathrm{rami}-$ pril-treated SHRSP, $23.1 \pm 2.7 \mathrm{ng} / \mathrm{ml} / \mathrm{hr}$ ).

As stated earlier, the only significant difference when comparing the peak responses to AII among the animal groups is found in the untreated WKY group. It is interesting to note, however, that when the phasic response to AII is normalized to the respective peak responses, the difference between the treated and untreated WKY groups is not present. Further, the alteration in phasic response in the SHRSP groups is only apparent when the values are normalized to peak response values. We therefore interpret the variation in peak response in the untreated WKY animal to be representative of a variation in crosssectional area or other similar factor that, when interpreted in isolation, is not significant, but rather functions as a normalization standard for other values so that "real" differences may be observed.

The enhanced contractile responsiveness in hypertension also involves an adrenergic component. Responsiveness to the adrenergic agonist, phenylephrine, was taken as a reflection of this adrenergic component of hypertension. Figure 4 illustrates the concentration-response curves to phenylephrine in carotid arteries from treated and untreated SHRSP and WKY rats. Panel A shows the cumulative concentration response curve to $\mathrm{PE}$ in carotid arteries from
WKY groups. Treatment during development did not significantly alter sensitivity to PE among adult WKY rats, though there was a significant difference between WKY groups and the untreated SHRSP group (on the order of one log unit; indicated by the dotted line; replotted data from right-hand panel). Panel B shows the cumulative concentration-response curve to PE in carotid arteries from SHRSP groups. Treatment during development with hydral/HCTZ did not significantly affect responsiveness in adult SHRSP. Treatment during development with ramipril, however, significantly shifted the concentration-response curve to the right in the adult SHRSP, yielding a curve that was characteristic of the responses from WKY groups. Threshold values to PE were taken as a measure of altered vascular responsiveness. In this case, the threshold value was defined as the concentration of $\mathrm{PE}$ that produced $10 \%$ of the maximal response to $\mathrm{PE}\left(\mathrm{EC}_{10}\right)$. Figure 5 shows the threshold $\left(\mathrm{EC}_{10}\right)$ for PE-induced contractions in the carotid artery from all animal groups. Carotid arteries from SHRSP showed a greater sensitivity to PE (lower $\mathrm{EC}_{10}$ ) than carotid arteries from WKY rats. Treatment with hydral/HCTZ did not significantly alter sensitivity to $\mathrm{PE}$ in carotid arteries from either strain. Threshold values to PE in carotid arteries from ramipril-treated rats, however, were significantly higher than values from untreated SHRSP but were not significantly different from WKY levels. Thus, treatment with ramipril prevented the development of increased sensitivity to PE in SHRSP.

Figure 6 examines the relationship between terminal blood pressure and vascular reactivity. Weighted linear regression analysis using all animal groups was used to calculate correlations between blood pressure and vascular reactivity. Figure $6 \mathrm{~A}$ illustrates the rela-
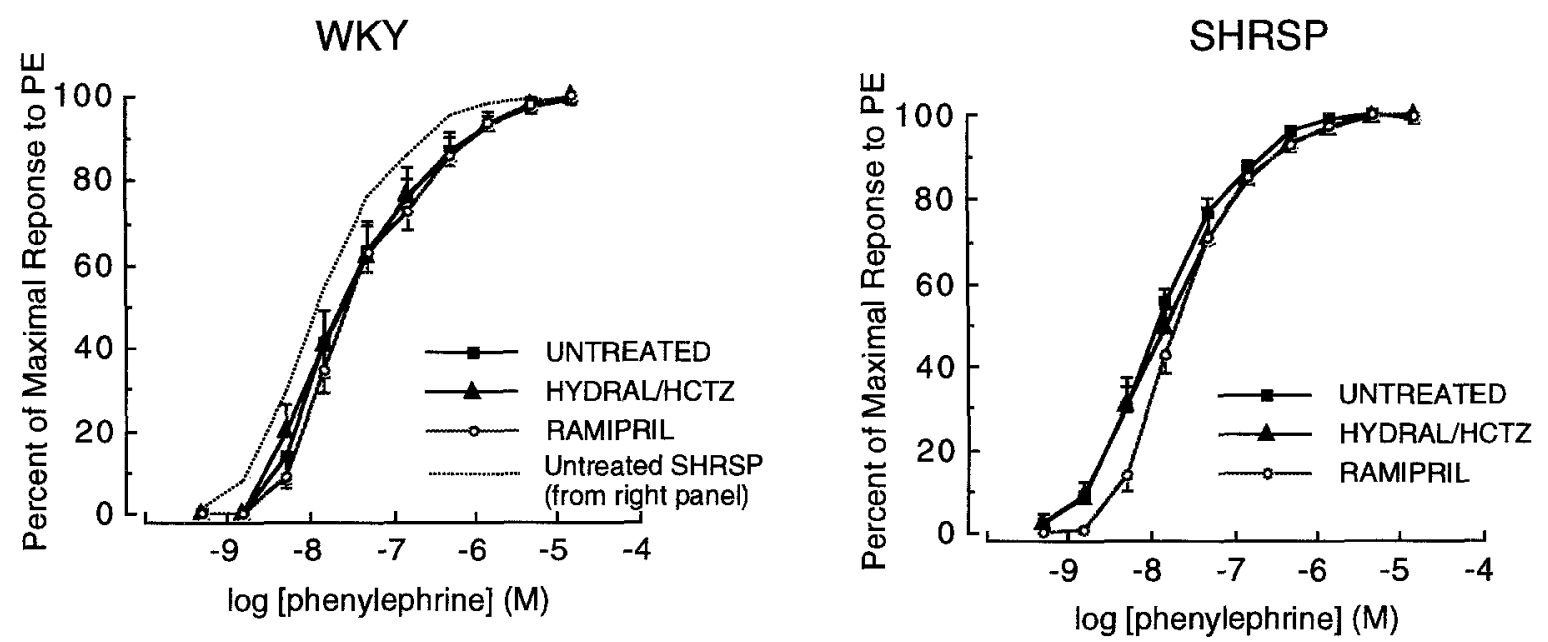

Fig. 4. Concentration-response curve to phenylephrine in carotid arteries from WKY and SHRSP control and treated rats. Cumulative concentrations of phenylephrine were introduced into the bath. Five-minute intervals were maintained between doses. $n$ values: WKY control (7), WKY hydralazine/HCTZ (6), WKY ramipril (8), SHRSP control (8), SHRSP hydralazine/HCTZ (5), SHRSP ramipril (8). * ${ }^{*}<.05$ by Student's $t$ test. 


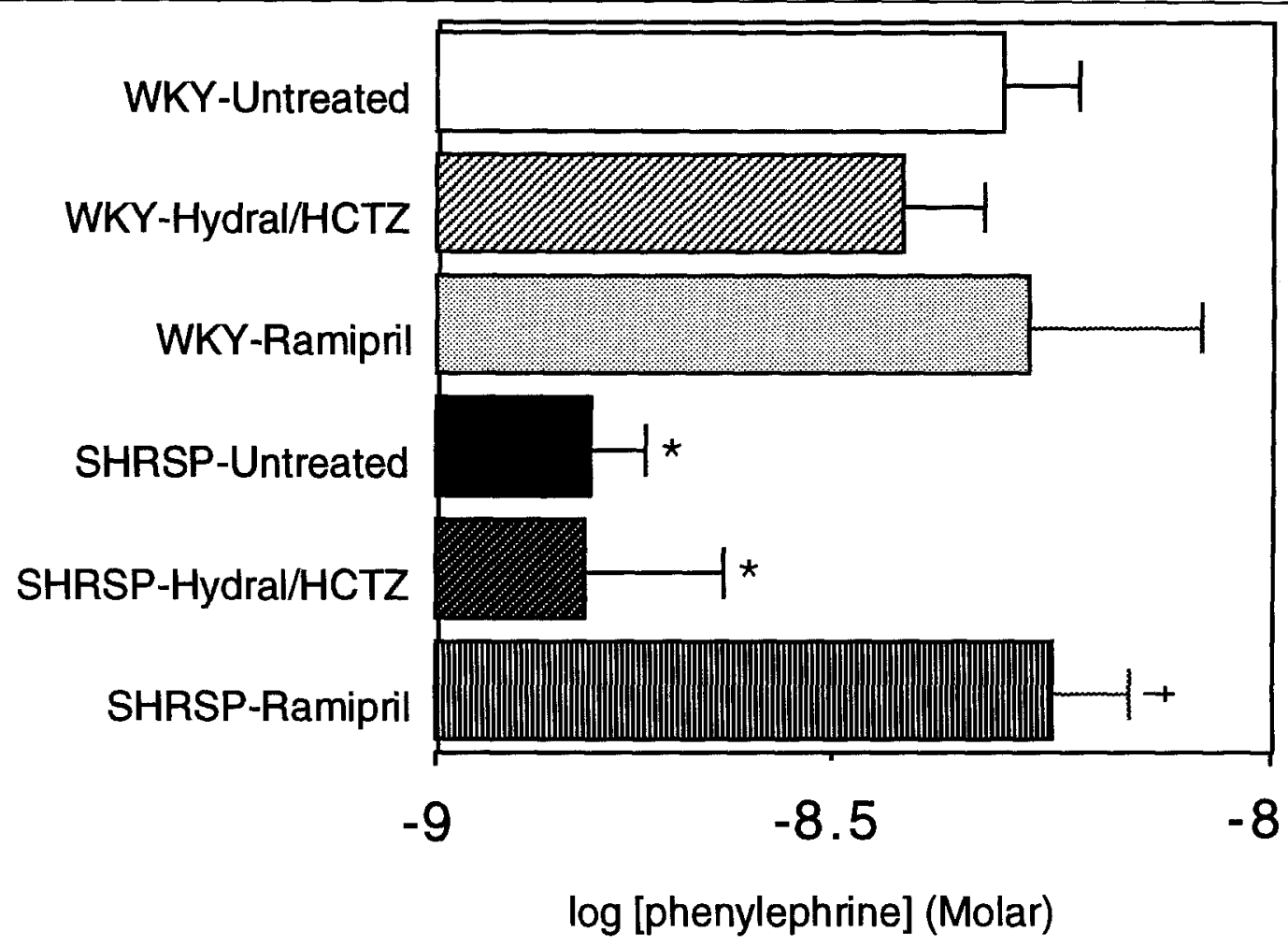

Fig. 5. $E C_{10}$ threshold values of phenylephrine-induced contraction in carotid arteries from SHRSP and WKY rats. Average concentration of $P E$ that elicits a contractile response equal to $10 \%$ of maximal contraction. $E C_{10}$ values were determined following graphical representation of normalized concentration-response curves. Data are expressed as means $\pm S E M$ values. $n$ values: WKY control (7), WKY hydralazine/HCTZ (6), WKY ramipril (8), SHRSP control (8), SHRSP hydralazine/HCTZ (5), SHRSP ramipril (8). Multiple $t$ tests with Bonferonni correction were used to determine statistical significance. ${ }^{*} p<.01$ when compared with the untreated WKY group. $t p<.01$ when compared with the untreated SHRSP group.

tionship between contractile responsiveness to $0.1 \mu \mathrm{M}$ AII in thoracic aorta and systolic blood pressure. Analysis revealed that the ramipril-treated SHRSP point did not significantly influence the slope of the correlation line. Figure $6 \mathrm{~B}$ illustrates the relationship between threshold values to $\mathrm{PE}$ in carotid arteries and systolic blood pressure. Weighted linear regression revealed that the ramipril-treated SHRSP point significantly influenced the slope of the correlation line; this point does not belong on the correlation line.

\section{Discussion}

The present study confirms previous work demonstrating that treatment with an ACE inhibitor early in the development of the genetically hypertensive animal prevents the full expression of hypertension in adulthood [6-10]. Changes in systolic blood pressure and vascular responsiveness were observed 7 weeks after cessation of the ACE inhibitor treatment. Contractile responsiveness to AII, shown to be altered in the hypertensive animal, was not significantly affected by treatment with ramipril but was correlated with terminal systolic blood pressure. Therefore, contractile responsiveness to AII does not appear to be associated with an AII-mediated genetic program in SHRSP and may be secondary to changes in blood pressure. In contrast, sensitivity to $\mathrm{PE}$ in the ramipril-treated SHRSP was not significantly different from WKY, and this change in sensitivity did not correlate with blood pressure. These results support a role for an AII-sensitive mechanism that may affect the development of genetic hypertension.

In terms of blood pressure development, it was clear that both ramipril and hydral/HCTZ were effective in reducing systolic blood pressure in both the WKY and SHRSP during the treatment period. After cessation of the treatment, however, the blood pressures of both treated WKY groups approached those of the untreated WKY, and at 17 weeks of age there was no significant difference among the WKY animals. Thus, antihypertensive treatment during the putative "critical period" in the normotensive animal does not seem to interfere with normal blood pressure development. After cessation of treatment in the SHRSP groups, the blood pressures of the hydral/ HCTZ-treated animals approached untreated SHRSP 

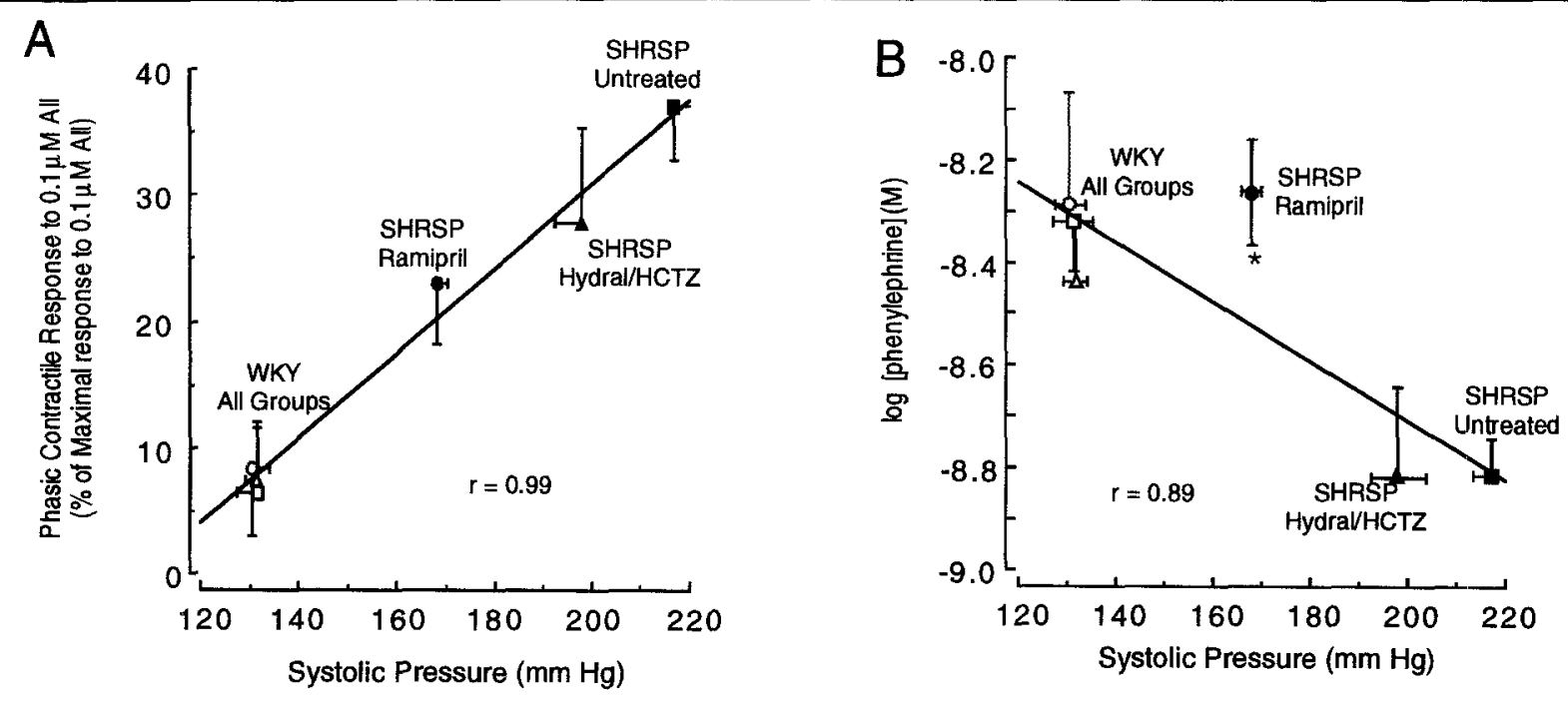

Fig. 6. Correlation between vascular responsiveness and average terminal blood pressure from treated and untreated WKY and SHRSP. A plots the correlation between contractile responsiveness to $0.1 \mu M$ Angiotensin II (AII) in thoracic aorta and terminal systolic blood pressure. A linear regression was performed using all groups, and it was determined that a positive correlation exists between blood pressure and contractile responsiveness to $0.1 \mu M A I I$. None of the groups lie significantly off the linear regression. B plots the correlation between $E C_{10}$ threshold values to phenylephrine in carotid arteries and average terminal blood pressure. A linear regression was performed using all points, and it was determined that a positive correlation exists between blood pressure and vascular responsiveness to phenylephrine. Only ramipril-treated SHRSP lie significantly off the linear regression. All data are expressed as means \pm SEM values. $n$ values: WKY untreated (7), WKY hydralazine/HCTZ (6), WKY ramipril (8), SHRSP untreated (8), SHRSP hydralazine/HCTZ (5), SHRSP ramipril (8).

blood pressure levels though at 17 weeks of age, and there was still a significant difference. In contrast, the blood pressures of the ramipril-treated SHRSP animals plateaued significantly earlier and remained approximately $50 \mathrm{mmHg}$ lower then the untreated SHRSP at 17 weeks of age. Clearly, simple reduction of blood pressure through the use of hydral/HCTZ during development did not account for the disparity between the untreated and rampril-treated SHRSP. Further, changes in body weight cannot account for this effect, as there were no significant differences in body weight among WKY and SHRSP. Rather, the chronic blood pressure-lowering effect of ramipril appeared to be the result of alterations in an AIIsensitive mechanism during a critical period of development. This conclusion is supported by the observation of Harrap et al. [6] that administration of AII along with ACE inhibitors resulted in the full expression of genetic hypertension. The question remains, however, as to what changes AII is effecting in the cardiovascular system in order to produce this antihypertensive effect.

One possible mechanism is alterations of the angiotensin-renin system. Intuitively, it might be expected that long-term changes associated with early ACE inhibition would affect responsiveness to AII in vascular smooth muscle or alter circulating AII levels. Previous studies have demonstrated an increased contractile responsiveness to AII in SHR through greater postsynaptic effects of the peptide [13], greater pressor [14] and slow-pressor [15] response to AII in SHR, and greater AII-stimulated increases in intracellular calcium [16]. Further, long-term treatment with ACE inhibitors has been shown to cause alterations in the renin-angiotensin system both centrally and peripherally that were manifested by changes in receptor binding and responsiveness to AII [17]. In this study we observed an increased contractile responsiveness in SHRSP to AII; however, these results were not significantly affected by ACE inhibitor treatment. Blocking AII generation during development does not appear to have any long-term effect on contractile responsiveness to AII. Further, plasma renin activity was not significantly different among the study groups. Hence, the target molecule for the long-term effects of ACE inhibition is unlikely to be AII. The increased responsiveness to AII in SHRSP, therefore, may be mediated by another genetic program that is regulated by some factor other than AII. Another possibility is that the increased responsiveness is secondary to the elevated blood pressure in SHRSP versus WKY. A second possible mechanism through which AII may modulate blood pressure is increased vascular smooth muscle growth. Investigators report that AII may mediate smooth muscle cell hypertrophy through changes in cytoskeletal $[18,19]$ and growth factors [20]. While structural alterations in vascular smooth muscle were not examined in the present 
study, recent reports suggest that vascular structure is a poor predictor of long-term hypertensive levels in SHR [9].

A third possibility is that reduced bradykinin degradation, which also occurs with administration of ACE inhibitors, may be partially responsible for the long-term effects of ACE inhibitors on blood pressure and vascular reactivity. Studies performed by Harrap et al. [6], however, show that long-term effects of antihypertensive treatment were not present when AII was coadministered with the ACE inhibitor. Further, persistent effects were also observed when the AII antagonist, losartan, was administered to hypertensive rats [9]. Therefore, it seems unlikely that bradykinin plays a major role in this phenomenon.

A fourth possible target for AII is the adrenergic response of vascular smooth muscle in hypertension. Studies have shown that AII can augment both the presynaptic and postsynaptic response to norepinephrine [13,21]. Draper et al. [22] report that AII enhanced the responses to peripheral nerve stimulation in preparations from normotensive and hypertensive animals, and that the enhancement was significantly greater in preparations from hypertensive animals. The present study characterized differences in sensitivities to phenylephrine in WKY and SHRSP. Treatment of SHRSP with ramipril prevented the development of increased sensitivity to phenylephrine that is characteristic of genetic hypertension. Moreover, the sensitivity to PE of the ramipril-treated SHRSP was not significantly different from those of the WKY groups. The sensitivity to $\mathrm{PE}$ does not appear to be a secondary effect of the reduced blood pressure in the ramipril-treated rat because hydral/HCTZ does not alter sensitivity to $\mathrm{PE}$ despite lowering blood pressure. Because the blood pressure of the ramipriltreated SHRSP is intermediate between the pressures of the untreated-SHRSP and the WKY groups, an association with blood pressure would dictate that the sensitivity to $\mathrm{PE}$ of the ramipril-treated animal would lie intermediate between the two groups. However, as shown in Figure 6, the $\mathrm{EC}_{10}$ for the ramipriltreated SHRSP is neither intermediate between the two groups nor correlated with blood pressure. This is further supported by the observation that ramipriltreated SHRSP show intermediate contractile responses to AII that both correlate with terminal blood pressure. Based on these data, we conclude that sensitivity to $\mathrm{PE}$ is determined by a genetic program that is influenced by AII. Thus, altered sensitivity to PE through the prevention of the enhancement of peripheral nerve stimulation by AII reported by Draper may be partially responsible for the attenuation of blood pressure observed in the ACE inhibitor-treated SHRSP.

It has been suggested that changes in vascular responsiveness in those subjects undergoing antihypertensive treatment may simply be the result of lower blood pressure. A recent study by Harrap et al. [23] attempts to address this issue by coadministering aldosterone with the ACE inhibitor treatment in order to sustain high blood pressure during the critical period. The blood pressure-lowering effect is not seen in these animals, and Harrap interprets this as meaning the effects of Ang II are most likely dependent on its blood pressure-lowering ability. The results of our study directly refute this interpretation by illustrating that decreased blood pressure alone during the critical period is not sufficient to produce the longterm effect. However, as Harrap also suggests, it is possible that AII does not exert its effects through either direct trophic or blood pressure-lowering ability but rather may be due to its effects on electrolyte balance through mediators such as aldosterone.

Further, experimental observations from other laboratories indicate that ACE inhibitors have effects on vascular responsiveness to agonists that are independent of its blood pressure-lowering effect. In a study by Sada et al. [24], SHR treated with hydralazine did not show changes in vascular responsiveness that were seen in SHR treated with ACE inhibitors, despite both sets of rats having blood pressures that were comparable $(\sim 130 \mathrm{mmHg})$. Therefore, it appears that ramipril is exerting its effects on the vasculature by some other means than solely lowering blood pressure.

In a study by Harrap et al. [6], sensitivity of the mesenteric artery from ACE inhibitor-treated SHR to norepinephrine was not significantly different from untreated SHR levels. Based upon this observation, they concluded that differences in contractile responsiveness were the result of structural changes rather than changes in the sensitivities of smooth muscle cells to norepinephrine. The present study shows that there was an altered sensitivity to phenylephrine in the carotid arteries from SHRSP and WKY rats. Moreover, ACE inhibition during development of the SHRSP does indeed alter sensitivity to phenylephrine in carotid arteries to levels observed in the WKY carotid artery. These separate observations concerning sensitivity to catecholamines may be due to different properties of the respective blood vessles. Indeed, it has been shown that different vascular beds possess different structural and functional characteristics.

It is interesting to note that treatment with either hydralazine [25] or hydrochlorothiazide [26] have been shown to result in slightly higher plasma renin activity levels. Unfortunately, since plasma renin activity levels were not measured during the experimental preparation of the animals, any comment on whether the AII levels are actually elevated or unchanged are purely speculative. However, it is clear that the "all or none" effect on development of blood pressure, as it appears in the literature, is not present in the animals treated with HCTZ/hydralazine. This supports the hypothesis that some genetic element is called into play during the development of the hypertensive animal that appears to require AII in order to be active. 
It follows that a slight elevation of AII levels should not affect this genetic program.

In summary, this study demonstrates that (a) a portion of blood pressure in the adult SHRSP is under the control of an AII-mediated genetic program that is activated early in development; (b) phasic contractile responsiveness to AII is augmented in SHRSP and this vascular trait is not under the control of an AIImediated genetic program; and (c) sensitivity to phenylephrine is increased in the adult SHRSP and this vascular trait is under the control of an AII-mediated genetic program. These data support the hypothesis that vascular abnormalities in SHRSP arise from a particular genetic program that is initiated early in development and contributes to the full expression of hypertension in the adult animal.

\section{Acknowledgments}

This work was supported by a grant from the National Institutes of Health (HL-18575). Oren Traub was the recipient of a fellowship from the American Heart Association of Michigan during this study.

\section{References}

1. Harrap SB. Causes and effects of high blood pressure: A longitudinal approach to genetic cosegregational analysis. $J$ Cardiovasc Pharmacol 1988;12(Supp1 3):S99-S109.

2. Soubrier F, Houot AM, Jeunemaitre X, Plouin PF, Corvol $P$. Molecular biology as a tool for genetic research in hypertension: Application to the renin gene. J Cardiovasc Pharmacol 1988;12(Suppl 3):S155-S159.

3. Mulvany MJ, Korsgaard N. Correlations and otherwise between blood pressure, cardiac mass and resistance vessel characteristics in hypertensive, normotensive and hypertensive/normotensive hybrid rats. $J$ Hypertens 1983;1:235-244.

4. Laher I, Triggle C. Blood pressure, lanthanum-, and norepinephrine-induced mechanical response in thoracic aortic tissue. Hypertension 1984;6:700-708.

5. Rapp JP. A genetic locus (Hyp-2) controlling vascular smooth muscle response in spontaneously hypertensive rats. Hypertension 1982;4:459-467.

6. Harrap SB, Van der Merwe WM, Griffin SA, Macpherson $\mathrm{F}$, Lever AF. Brief angiotensin converting enzyme inhibitor treatment in young spontaneously hypertensive rats reduces blood pressure long term. Hypertension 1990;16: $603-616$.

7. Lee RM, Berecek KH, Tsoporis J, McKenzie R, Triggle $\mathrm{CR}$. Prevention of hypertension and vascular changes by captopril treatment. Hypertension 1991;17:141-150.

8. Harrap SB. A developmental genetic mechanism involving angiotensin in spontaneously hypertensive rats. Clin Exp Pharmacol Physiol Suppl 1992;19:19-22.

9. Morton JJ, Beattie EC, MacPherson F. Angiotensin II receptor antagonist losartan has persistent effects on blood pressure in the young spontaneously hypertensive rat: Lack of relation to vascular structure. $J$ Vasc Res 1992;29: 264-269.

10. Giudicelli JF, Freslon JL, Glasson S, Richer C. Captopril and hypertension development in the SHR. Clin Exp Hypertens 1980;2:1083-1096.

11. Sessler FM, Kim SH, Malvin RL. Changes of renin isoelectric heterogeneity after acute and chronic stimulation of renin secretion. Proc Soc Exp Biol Med 1986;182:258-262.

12. Kojima M, Aoki K, Fujimoto S, Matsoda T. Malfunction of arterial sarcoplasmic reticulum leading to faster and greater contraction induced by high-potassium depolarization in young spontaneously hypertensive rats. $J$ Hypertens $1991 ; 9$ : 783-788.

13. Urabe M, Su, Lee TJ. Pre- and postsynaptic effects of angiotensins in the femoral artery of spontaneously hypertensive and Wistar-Kyoto rats. Blood Vessels 1987;24:1-10.

14. Couture R, Regoli D. Vascular reactivity to angiotensin and noradrenaline in spontaneously and renal hypertensive rats. Clin Exp Hypertens 1980;2:45-63.

15. Li P, Jackson EK. Enhanced slow-pressor response to angiotensin II in spontaneously hypertensive rats. J Pharmacol Exp Ther 1989;251:909-921.

16. Bendhack LM, Sharma RV, Bhalla RC. Altered signal transduction in vascular smooth muscle cells of spontaneously hypertensive rats. Hypertension 1992;19(Suppl): II142-II148.

17. Wilson KM, Magargal W, Berecek KH. Long-term captopril treatment. Angiotensin II receptors and responses. Hypertension 1988;11:I148-I152.

18. Owens GK. Role of contractile agonists in growth regulation of vascular smooth muscle cells. Adv Exp Med Biol 1991; 308:71-79.

19. Mackie EJ, Scott-Burden T, Hahn AW, et al. Expression of tenascin by vascular smooth muscle cells. Alterations in hypertensive rats and stimulation by angiotensin II. $A m J$ Pathol 1992;141:377-388.

20. Paul M, Ganten D. The molecular basis of cardiovascular hypertrophy: The role of the renin-angiotensin system. $J$ Cardiovasc Pharmacol 1992;19(Suppl 5):S51-S58.

21. Bohr DF, Uchida E. Individualities of vascular smooth muscles in response to angiotensin. Cire Res 1967;21(Suppl 2): 135 .

22. Draper AJ, Meghji S, Redfern PH. Enhanced presynaptic facilitation of vascular adrenergic neurotransmission in spontaneously hypertensive rats. I Auton Pharmacol 1989; 9:103-111.

23. Harrap SB, Mitchell GA, Casley DJ, Mirakian C, Doyle AE. Angiotensin II, sodium, and cardiovascular hypertrophy in spontaneously hypertensive rats. Hypertension 1993;21: $50-55$.

24. Sada T, Koike H, Miyamoto M. Long-term inhibition of angiotensin converting enzyme suppresses calcium channel agonist-induced contraction of aorta in hypertensive rats. $\mathrm{Hy}$ pertension 1989;14:652-659.

25. Tofovic SP, Branch KR, Oliver RD, Magee WD, Jackson EK. Caffeine potentiates vasodilator-induced renin release. $J$ Pharmacol Exp Ther 1991;256:850-860.

26. Luft FC, Fineberg NS, Weinberger MH. Long-term effect of nifedipine and hydrochlorothiazide on blood pressure and sodium homeostasis at varying levels of salt intake in mildly hypertensive patients. Am J Hypertens 1991;4:752-760. 